\title{
NOTE ON THE CURVATURE OF ORTHOGONAL TRAJECTORIES OF LEVEL CURVES OF GREEN'S FUNCTIONS
}

\section{J. L. WALSH}

The writer has recently established the following theorem :*

TheOREM I. Let $R$ be a simply connected region of the extended $(x, y)$ plane whose boundary $B$ contains at least two points. Let $G(x, y)$ be Green's function for $R$ with pole in the point $O$. Let $\{T\}$ denote the set of orthogonal trajectories to the level curves $G(x, y)=\log r,(0<r<1)$. The totality of circles each osculating a curve $T$ at $O$ is precisely the set of circles through $O$ and through another fixed point $D$ depending on $O$ and $R$. There exists no circle that separates both $O$ and $D$ from $B$.

The curves $T$ are of course the images of the radii under the conformal mapping of a circle onto $R$, so that the center of the circle corresponds to $O$.

In Theorem I, the term "circle" is used in the extended sense, to include straight lines.

In the situation of Theorem I, we shall call $D$ the conjugate of $O$ with respect to $R$. This terminology seems justified, because in the case that $B$ is a line, the point $D$ is the reflection of $O$ in $B$; and in the case that $B$ is a proper circle, the point $D$ is the inverse of $O$ in $B$.

It is the object of the present note to establish the following theorem:

THEOREM II. Under the hypotheses of Theorem I the point D may be chosen arbitrarily exterior to $R$; if $D$ is so chosen there exists at least one point $O$ interior to $R$ whose conjugate with respect to $R$ is $D$.

In the proof of Theorem II it is sufficient, by the use of a suitably chosen linear transformation, to treat the special case that $D$ is chosen at infinity. $\dagger$

THEOREM III. Let $R$ be an arbitrary limited simply connected region $R$. There exists interior to $R$ at least one point $O$ whose conjugate $D$ lies at infinity. That is to say, there exists at least one point $O$ of $R$ such that the orthogonal trajectories to the level curves of Green's function with pole at $O$ have at $O$ zero curvature.

* Proceedings of the National Academy of Sciences, vol. 23 (1937), pp. 166-169.

$\dagger$ Choice of $O$ at infinity yields the theorem: Let $R$ be a simply connected region, and let $D$ be a point exterior to $R$. There exists a linear transformation of the plane which carries $R$ into an infinite region for which the image of $D$ is the conformal center of gravity. 
The proof of Theorem III is to be set forth with the aid of several lemmas. If $R$ is a limited simply connected region of the z-plane, the inner radius of $R$ with respect to an interior point $O: z=a$ is defined as the unique positive number $r(a)$ such that $R$ can be mapped conformally onto the region $|w|<r(a)$ by a function of the form

$$
z-a=w+c_{2} w^{2}+c_{3} w^{3}+\cdots, \quad|w|<r(a) .
$$

LEMMA I. If $R$ is a limited simply connected region of the z-plane, if $O: z=a$ lies interior to $R$, and if $\delta(a)$ denotes the distance from $O$ to the boundary of $R$, then we have

$$
4 \delta(a) \geqq r(a) .
$$

Let the function (1) map $R$ onto $|w|<r(a)$. Then the function

$$
\zeta=\frac{1}{r(a)}\left[r(a) \omega+c_{2}[r(a)]^{2} \omega^{2}+c_{3}[r(a)]^{3} \omega^{3}+\cdots\right], \quad|\omega|<1,
$$

maps the region $R_{1}$ of the $\zeta$-plane, obtained from $R$ by the transformation $\zeta=(z-a) / r(a)$, onto the region $|\omega|<1$. The distance from the point $\zeta=0$ to the boundary of $R_{1}$ is $\delta(a) / r(a)$, so inequality (2) follows at once from the Verzerrungssatz.* An immediate conclusion is the following lemma:

LEMMA II. Under the hypothesis of Lemma I the function $r(a) a p$ proaches zero whenever the point $O: z=a$ approaches the boundary of $R . \dagger$

* See for instance Pólya-Szegö, Aufgaben und Lehrsätze aus der Analysis, vol. 2, Berlin, 1925, p. 27 , exercise 152 . Lemma II is given there explicitly (p. 19, exercise 112 ) for the case that $R$ is bounded by an analytic curve.

$\dagger$ Let the function $z=f(w)$ map conformally $|w|<1$ onto $R$, with $a=f(b)$; then the function $z=\phi(w)=f[(w+b) /(1+\bar{b} w)]$ maps $|w|<1$ onto $R$ with $a=\phi(0)$, and we have $r(a)=\left|\phi^{\prime}(0)\right|=\left|f^{\prime}(b)\right|\left(1-|b|^{2}\right)$, so Lemma $I$ is precisely the inequality

$$
4 \delta(a) \geqq\left|f^{\prime}(b)\right|\left(1-|b|^{2}\right) ;
$$

Lemma II asserts precisely the conclusion

$$
\text { (A) } \quad \lim \left|f^{\prime}(b)\right|\left(1-|b|^{2}\right)=0 \text {, }
$$

as $a$ approaches the boundary of $R$ (or, what is the same thing, as $|b| \rightarrow 1$ ).

Still another method of proof of Lemma II is the following. Instead of keeping the region $R$ fixed and allowing a sequence of interior points $z=a$ to approach the boundary of $R$, we may study $r(a)$ by keeping the point $z=a$ fixed and allowing the region $R$ to vary by a sequence of translations in such a way that the boundary of the variable $R$ approaches $z=a$. Again by the use of $\phi(w)$, Carathéodory's theory of the conformal mapping of variable regions then yields Lemma II.

These two distinct methods in connection with the study of $\phi(w)$, namely (i) use of various inequalities such as (2), and (ii) Carathéodory's theory of variable regions, can be employed not merely for the proof of (A) but can also be used to study higher derivatives of $f(w)$, as will be shown on another occasion. 
The following lemma will also be useful:

Lemma III. If $r(a)$ has a relative maximum at the point $O:|z|=a$, then for the corresponding mapping function (1) we have $c_{2}=0$, and the conjugate of $O$ lies at infinity.

Under the present hypothesis the relation $a_{2}=0$ is not difficult to establish,* where the inverse of (1) is given by

$$
w=(z-a)+a_{2}(z-a)^{2}+a_{3}(z-a)^{3}+\cdots .
$$

By the usual formulas for the inversion of the power series (1), we then have $c_{2}=0$, from which it follows $\dagger$ that the conjugate of $O$ lies at infinity.

We are now in a position to establish Theorem III. The function $r(a)$ is positive and continuous $\ddagger$ at each point $O: z=a$ of $R$. Whenever the point $O$ in $R$ approaches the boundary of $R$, the function $r(a)$ approaches zero, by Lemma II. The function $r(a)$, when suitably defined on the boundary, is then continuous in the corresponding closed region and possesses an absolute maximum in that region. That maximum occurs interior to $R$ and is necessarily a relative maximum of $r(a)$. Theorem III follows from Lemma III.

We now give an example to show that when $D$ is given the point $O$ of which $D$ is the conjugate need not be unique. For convenience we choose the situation of Theorem III. Let $R$ be the region of the $z$ plane formed by the interiors of the two circles

$$
\left|z \pm(.99)^{1 / 2}\right|=1
$$

the two points $z= \pm i / 10$ lie on the boundary of $R$. In the notation already introduced, we have $r(0) \leqq .4$, by Lemma I. But $R$ contains in its interior the region $\left|z-(.99)^{1 / 2}\right|<1$, so we have $r(.99)^{1 / 2} \geqq 1$. Thus the point $z=0$ does not furnish an absolute maximum for the function $r(a)$; such an absolute maximum exists interior to $R$; such an absolute maximum cannot be unique, for if $z=\alpha$ furnishes an absolute maximum, so also does $z=-\alpha \neq \alpha$; and every such absolute maximum has its conjugate at infinity.

In the example just given, the point $z=0$ has its conjugate at infinity even though it does not give an absolute maximum to the function $r(a)$.

* Pólya-Szegö, op. cit., p. 19, exercise 113.

$\dagger$ Walsh, loc. cit.

$\ddagger$ The continuity follows for instance from the formula for $r(a)$ in terms of a fixed $r(b)$; Pólya-Szegö, op. cit., p. 19, exercise 110.

$\S$ Pólya-Szegö, op. cit., p. 21, exercise 121. 
A slight modification of the example given shows that if $N$ is chosen arbitrarily, there exists a limited region* having at least $N$ distinct points $O$ whose conjugates $D$ lie at infinity. $\dagger$

Theorem III becomes false if in the hypothesis the region $R$ is not assumed limited, for the reader may verify that no point $O$ of the region $R$ has its conjugate at infinity if $R$ is the entire plane slit along the positive half of the axis of reals from the point $z=0$ to infinity.

HARVARD UNIVERSITY

\section{A NOTE ON LINEAR FUNCTIONALS}

\section{R. P. BOAS, JR., $\ddagger$ AND J. W. TUKEY}

1. Introduction. The knowledge of the general form of linear functionals in a given abstract space $\S$ is of value in many problems. In some cases (notably in the theory of moment problems) the applications are not to the given space, but to its conjugate space; for example, since the general linear functional on $L$, the space of functions $x=x(t)$ integrable on $(0,1)$, has the form

$$
f(x)=\int_{0}^{1} x(t) a(t) d t, a \text { measurable and essentially bounded, } \|
$$

one can solve the moment problem

$$
\mu_{n}=\int_{0}^{1} t^{n} a(t) d t, \quad n=0,1,2, \cdots,
$$

for essentially bounded functions $a . \|$ From the point of view of the theory of moment problems, it seems quite fortuitous that there

\footnotetext{
* For the unlimited region $R:|y| \leqq b>0$ of the $(x, y)$-plane, every point $(x, 0)$ has as conjugate the point at infinity.

$\dagger$ The referee points out that for any region the set of points $O$ whose conjugates $D$ lie at infinity is identical with the set of critical points of the function $r(a)$.

$\ddagger$ National Research Fellow.

$\S$ We use the terminology of S. Banach, Théorie des Opérations Linéaires, Warsaw, 1932.

\| S. Banach, op. cit., p. 65. The function $a$ is said to be essentially bounded if there is a number $M$ such that $|a(t)| \leqq M$ for almost all $t$ on $(0,1)$; we denote by $\sup ^{0}|a(t)|$ the greatest lower bound of such numbers $M$.

I S. Banach, op. cit., p. 75.
} 